\title{
Discovery of 20 novel ribosomal leader candidates in bacteria and archaea
}

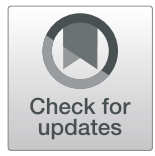

Iris Eckert and Zasha Weinberg ${ }^{*}$ (1)

\begin{abstract}
Background: RNAs perform many functions in addition to supplying coding templates, such as binding proteins. RNA-protein interactions are important in multiple processes in all domains of life, and the discovery of additional protein-binding RNAs expands the scope for studying such interactions. To find such RNAs, we exploited a form of ribosomal regulation. Ribosome biosynthesis must be tightly regulated to ensure that concentrations of rRNAs and ribosomal proteins (r-proteins) match. One regulatory mechanism is a ribosomal leader (r-leader), which is a domain in the $5^{\prime}$ UTR of an mRNA whose genes encode r-proteins. When the concentration of one of these r-proteins is high, the protein binds the r-leader in its own mRNA, reducing gene expression and thus protein concentrations. To date, 35 types of $r$-leaders have been validated or predicted.

Results: By analyzing additional conserved RNA structures on a multi-genome scale, we identified 20 novel r-leader structures. Surprisingly, these included new r-leaders in the highly studied organisms Escherichia coli and Bacillus subtilis. Our results reveal several cases where multiple unrelated RNA structures likely bind the same r-protein ligand, and uncover previously unknown r-protein ligands. Each r-leader consistently occurs upstream of r-protein genes, suggesting a regulatory function. That the predicted r-leaders function as RNAs is supported by evolutionary correlations in the nucleotide sequences that are characteristic of a conserved RNA secondary structure. The rleader predictions are also consistent with the locations of experimentally determined transcription start sites.

Conclusions: This work increases the number of known or predicted r-leader structures by more than $50 \%$, providing additional opportunities to study structural and evolutionary aspects of RNA-protein interactions. These results provide a starting point for detailed experimental studies.
\end{abstract}

Keywords: Comparative genomics, Ribosomal leader, Bioinformatics, RNA-protein interaction, cis-regulatory RNA

\section{Background}

The ribosome is an RNA-protein complex that performs protein synthesis in all living cells [1-3]. The ribosome consists of two subunits: the small subunit binds the mRNA template, while the large subunit catalyzes the peptidyl transfer reaction. Each bacterial or archaeal ribosome is made of three different rRNAs (5S, $16 \mathrm{~S}$ and 23S) and many ribosomal proteins (r-proteins). Ribosome synthesis is a complex process involving multiple

* Correspondence: zasha@bioinf.uni-leipzig.de

Bioinformatics Group, Department of Computer Science and Interdisciplinary Centre for Bioinformatics, Leipzig University, Härtelstraße 16-18, 04107 Leipzig, Germany

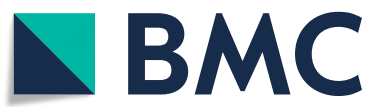

maturation steps, including the processing and folding of rRNA through the binding of r-proteins.

Because of the central importance of the ribosome to cellular function, ribosomes consume a large portion of the cell's energy [4]. As a result of this huge cost in energy, cells use highly optimized regulatory systems to ensure that $r$-proteins are at their optimal concentrations [4].

One common regulatory system in bacteria is a type of feedback regulation known as a ribosomal leader ( $\mathrm{r}$ leader) [4-8]. R-leaders are structured RNA elements that occur in the $5^{\prime}$ UTRs of mRNAs whose genes encode r-proteins. One or more of these r-proteins can interact with the r-leader, in addition to the r-protein's

(c) The Author(s). 2020 Open Access This article is licensed under a Creative Commons Attribution 4.0 International License, which permits use, sharing, adaptation, distribution and reproduction in any medium or format, as long as you give appropriate credit to the original author(s) and the source, provide a link to the Creative Commons licence, and indicate if changes were made. The images or other third party material in this article are included in the article's Creative Commons licence, unless indicated otherwise in a credit line to the material. If material is not included in the article's Creative Commons licence and your intended use is not permitted by statutory regulation or exceeds the permitted use, you will need to obtain permission directly from the copyright holder. To view a copy of this licence, visit http://creativecommons.org/licenses/by/4.0/ The Creative Commons Public Domain Dedication waiver (http://creativecommons.org/publicdomain/zero/1.0/) applies to the data made available in this article, unless otherwise stated in a credit line to the data. 
normal role in the ribosome. Excess r-proteins bind the r-leader, which leads to a change in the 5' UTR's secondary structure that results in repressed expression of the downstream genes. Known mechanisms for repression [7] include the conditional formation of Rhoindependent transcription terminators and sequestration of the ribosome-binding site.

Because the $r$-protein ligands of $r$-leaders also bind rRNA, it was hypothesized that r-leaders imitate the structure of the relevant rRNA binding site $[5,7]$. In several cases, this similarity is apparent from the secondary structure [9-11], while other cases require a crystal structure to demonstrate structural mimicry $[12,13]$. However, it is not a requirement that the $r$ leader must copy the rRNA structure; in a few cases, no meaningful similarities could be detected [7].

Thirty-five r-leaders have been confirmed or proposed (Additional file 1: Table S1) [7]. Knowledge of r-leaders is important to provide a complete picture of ribosome assembly and overall cellular metabolism.

R-leaders also provide a starting point for deeper research into the structure and evolution of RNAprotein interactions [14], which are important in all domains of life in a variety of contexts. For example, some $r$-proteins are the ligand of multiple types of $r$ leaders that each have different conserved sequence and structural features. These distinct structures thus exhibit multiple solutions to a single biochemical problem, and create opportunities [15] to study the evolutionary and structural influences leading to these end-points.

Additionally, r-leaders can be a model system to understand cis-regulatory mechanisms. For example, only a handful of cis-regulatory RNAs in archaea are experimentally confirmed or even predicted [16, 17]. Information on this aspect of gene regulation is thus lacking.

We therefore decided to detect novel $r$-leaders using a bioinformatics strategy centered around a phenomenon known as covariation. Covariation typically refers to mutations in which both nucleotides involved in a Watson-Crick base pair change to form a different Watson-Crick base pair (or the compatible G-U base pair). With sufficient evolutionary time, such mutations are frequent in RNAs that conserve a structure, but only occur sporadically by chance in sequences that do not function as RNAs. Analysis of covariation has been highly successful in determining conserved structures of molecules, such as ribosomal RNAs, that were later confirmed experimentally [1820]. Covariation-based strategies have also been used to find RNAs de novo, which have resulted in experimentally validated riboswitches [21], ribozymes [22] and an r-leader [23, 24], among others.

\section{Results}

\section{Discovery and evaluation of candidate r-leaders}

To find novel r-leaders, we inspected raw computer predictions of conserved RNA secondary structures from earlier studies [25, 26]. Each prediction consisted of a multiple sequence alignment and a conserved secondary structure. We call these predictions "motifs". In searching for r-leaders, the most promising motifs are those that are frequently located upstream of genes that encode r-proteins, and are thus potential cis-regulators of these genes. We analyzed the 32 best predictions by finding additional homologs and exploiting covariation to find additional conserved RNA secondary structure, using previously established approaches [25-27].

After this analysis, motifs whose secondary structures were supported by covariation and that remained consistently upstream of genes encoding r-proteins were considered candidate r-leaders. The evaluation of covariation is often not straightforward [26] (see Methods). To ensure that none of our motifs were previously discovered, we compared them to previously established RNAs. We compiled a list of all $r$-leaders that are experimentally verified or have a predicted alignment (Additional file 1: Table S1). We eliminated candidate motifs whose homologs overlap previously published RNAs, and those whose primary and secondary structure features were essentially the same as any previously published $r$-leader (see Methods). However, we included a new S4 r-leader in Fusobacteria whose potential binding site resembles that of the previously published Firmicutes S4 r-leader, but occurs in the context of a different secondary structure. We thus report 20 novel r-leader motifs (Table 1, Fig. 1). The 20 new r-leaders show no meaningful similarities to one another (see Methods), except for a partial resemblance between the archaeal S15 leaders (see below). We refer to the new motifs (Table 1, Fig. 1) based on their most likely ligands and the lineage of bacteria or archaea in which they occur (e.g., the Fusobacteria S4 r-leader motif).

In addition to the 20 novel r-leaders, we found three motifs (Additional file 1: Figure S1) whose secondary structure and nucleotide conservation patterns are fundamentally the same as already-established motifs, but that included additional homologs not previously found. These were the L25 r-leader in Enterobacteria [28], the S10 rleader in Firmicutes [29], where we found numerous examples in the sub-lineage Clostridia, and the L19 leader in Firmicutes [29], where we found many examples in the distinct phylum Flavobacteria. These alignments are available as Additional files (see below), but are not further discussed in this manuscript, except for Fig. 1.

All novel motifs are summarized in Table 1. Statistics regarding base pairs and covariation are provided (Additional file 1: Table S2), and include statistically 
Table 1 Summary of novel ribosomal leaders

\begin{tabular}{|c|c|c|c|c|}
\hline Most likely ligand & Ligand basis & Regulated gene(s) & Lineage(s) & Motif rating \\
\hline L2 & Closest & $r p / B, r p s S, r p / V, r p s C$ & Alphaproteobacteria & $?$ \\
\hline L4 & Prior & $r p / C, \underline{r p / D}, r p / W, r p / B+$ & Archaeoglobi (Archaea) & $?$ \\
\hline L13 & Prior & $\underline{r p / M}, r p s l,+r p s B$ & Bacteroidia & Y \\
\hline eL15 * & Only & rpl15e & Euryarchaeota (Archaea) & Y \\
\hline L17 & Only & $r p / Q$ & Actino- and Proteobacteria & Y \\
\hline $\mathrm{L} 20$ * & Only & $\underline{r p s T}$ & Deltaproteobacteria & Y \\
\hline L31 & Only & rpmE & Actinobacteria & Y \\
\hline L31 & Only & rpmE & Coriobacteria & Y \\
\hline L31 & Prior@ & $r p m E, r p m F$ & Corynebacteriaceae & Y \\
\hline L31 & Only & rpmE & Firmicutes & Y \\
\hline L31 & Only & rpmE & Gammaproteobacteria & Y \\
\hline S4 & Prior & $\underline{r p s D}, r p \circ A, r p / Q$ & Bacteroidia & Y \\
\hline S4 & Prior & $r p s M, r p s K, \underline{r p s D}, r p o A, r p / Q$ & Clostridia & Y \\
\hline S4 & Prior & $\underline{r p s D}, r p \circ A, r p / Q$ & Flavobacteria & Y \\
\hline S4 * & Prior & $r p s M, r p s K, \underline{r p s D}, r p o A, r p / Q$ & Fusobacteriales & Y \\
\hline S6:S18 & Prior & $\underline{r p s F}, s s b A, \underline{r p s R}, r p l l$ & Chlorobi & Y \\
\hline S15 & Only & $\underline{r p s O}$ & Flavobacteria & $?$ \\
\hline $\mathrm{S} 15 *$ & Prior & $\underline{\mathrm{rpsO}},+\mathrm{rec}, \mathrm{rps} 3 a e$ & Halobacteria (Archaea) & Y \\
\hline $\mathrm{S} 15^{*}$ & Prior & $\underline{r p s O},+r e c,, r p s 3 a e$ & Methanomicrobia (Archaea) & Y \\
\hline S16 & Only & rpsP, rimM & Flavobacteria & Y \\
\hline
\end{tabular}

"Most-likely ligand": see text. All proteins are the bacterial version, except eL15 is the eukaryotic/archaeal "L15" protein. Some principles used for ligand predictions are uncertain (see text). Asterisk $\left(^{*}\right)$ : r-leader is potentially similar to rRNA binding site for the given ligand, supporting its assignment. "Ligand basis": principle with which the most likely gene was hypothesized. "Only": there is only one regulated r-gene; "Prior": there are multiple regulated genes and one encodes a known ligand of another r-leader (at sign (@): based on L31 motifs in the current paper), "Closest": the immediately downstream gene. "Regulated gene(s)": genes consistently located in potentially regulated operons are listed in the order they most often appear. Most genes encode ribosomal proteins. Genes encoding the ligand of a previously established r-leader are underlined. Motifs lacking underlined genes bind novel r-protein ligands. Plus sign: apparent operon can often be extended. (Potential additional genes are shown in three cases.) "Lineage": taxon containing the motif. Archaeal taxa are indicated. "Motif rating": covariation evidence supporting assignment as RNA. " $Y$ " = likely candidate, "?" = borderline candidate. The reasons for assigning candidates as borderline are given in Additional file 1: Supplementary text

significant covariation signals (see Methods) for all $20 \mathrm{r}$ leader motifs. For each motif, we also provide alignments and information on downstream genes and taxonomy (Additional file 2) and machine-readable alignments with (Additional file 3) and without (Additional file 4) additional metadata. We found some stems with very borderline support from covariation. These stems are indicated only in Additional files 2 and 3. Consensus diagrams for all novel motifs, previously published motifs that bind the same putative ligand, as well as the relevant protein-binding sites in rRNAs are found in Additional file 1: Figures S2-S11.

\section{Transcriptome data}

The above analysis was conducted without regard to information on which genomic locations are transcribed. As a result, it is possible that a predicted r-leader would be in a location that is not transcribed with the downstream gene, a finding that would suggest that the predicted r-leader does not regulate the gene in cis. This finding would thus contradict our hypothesis that the motif corresponds to an r-leader. We therefore retrieved studies that established transcription start sites (TSSes) based on high-throughput transcriptomics data. These data are consistent with our r-leader predictions (Additional file 1: Figure S12, Table S3, Supplementary text, sub-heading "Candidate r-leaders are consistent with available transcriptomic data").

\section{Analysis of ligands and binding sites}

To generate hypotheses for the r-protein ligands of the motifs, we assumed that the ligand would be encoded by a gene regulated by the r-leader, as this is true in all known cases. Eight novel r-leader motifs were observed only upstream of genes encoding one $r$-protein (Table 1 ), and in these cases we concluded that this $r$-protein is the ligand. A ninth motif is directly upstream of rpsP, which encodes r-protein S16, and also usually upstream of rimM genes, which are involved in 16S rRNA processing. We believe this motif likely functions as an S16 r-leader (Table 1) despite the rimM genes (explained in Additional file 1: Supplementary text, subheading "S16"). 


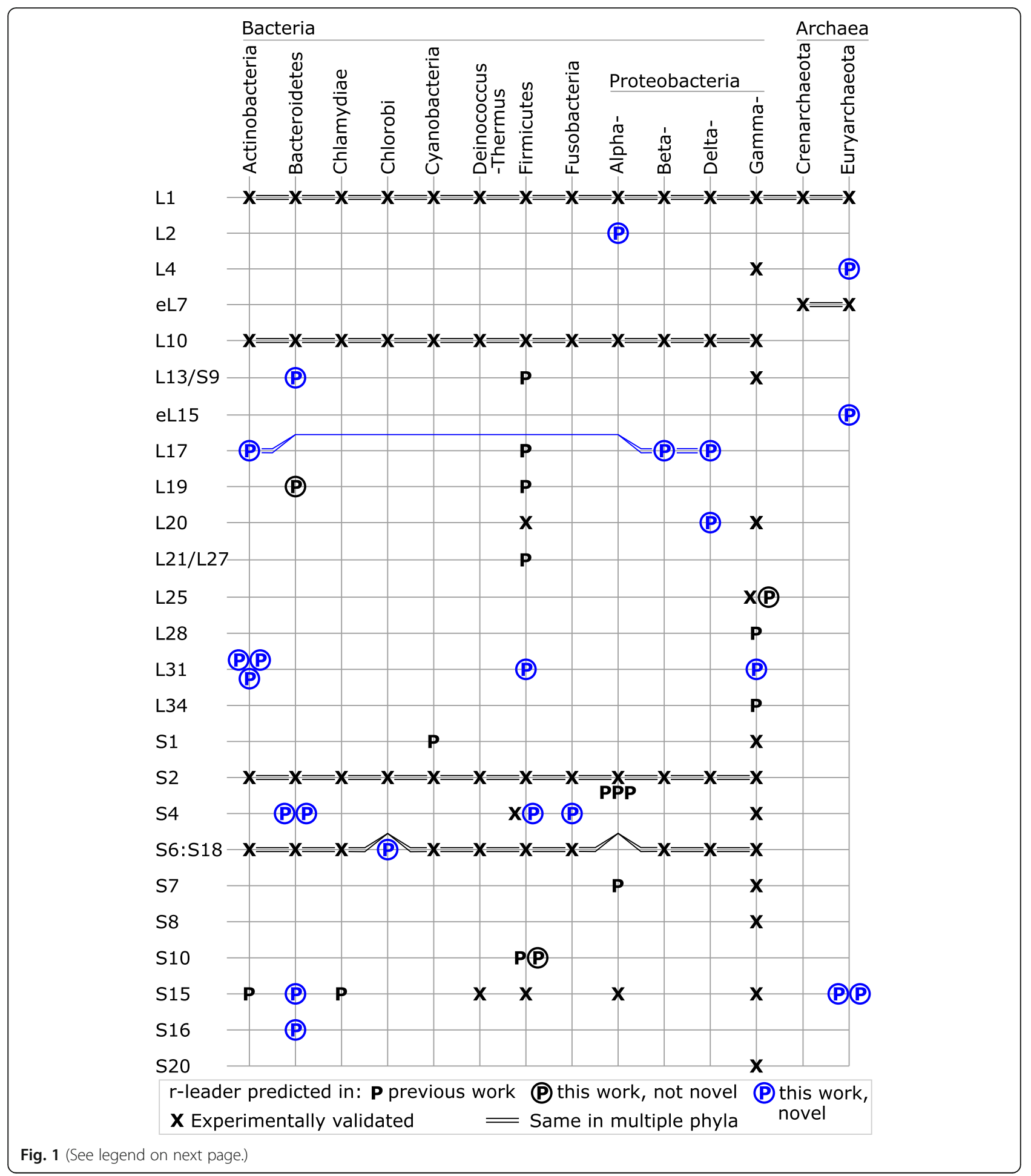


(See figure on previous page.)

Fig. 1 Occurrence of previously and newly found r-leaders in bacteria and archaea. R-leaders predicted or confirmed to bind various r-proteins are shown along with the phyla or classes in which they occur. R-leaders predicted in "this work, not novel" structurally resemble previously predicted r-leaders (Additional file 1: Figure S1). "same in multiple phyla": some r-leaders occur in more than one phylum. Multiple letters in a grid point imply multiple motifs, usually for different sub-lineages of the given phylum. eL7, eL15: r-proteins specific to eukaryotes and archaea. The ligands of predicted r-leaders have differing degrees of confidence (e.g., see Table 1), and could be incorrect. Phyla whose r-leaders are all present in other already-shown phyla are not listed, except that Crenarchaeota is depicted. The phyla containing previously identified r-leaders are determined based on previous reports (see Additional file 1: Table S1), and not based on homology searches, because established search parameters are lacking in most cases. Some r-leaders are only identified in a single species or a limited taxa; we depicted these r-leaders as present in the phyla. The figure suggests a conspicuous absence of a S6:S18 r-leader in Alphaproteobacteria; we see promising motifs that likely correspond to the missing r-leaders (Weinberg, unpublished data). The data in this figure are derived from Table 1, Additional file 1: Table S1 and the three motifs in Additional file 1: Figure S1

The remaining 11 motifs appear to regulate multiple r-protein genes (Table 1), so it is not possible to make as confident a prediction. It might seem most likely that the ligand would be encoded by the gene immediately downstream of the r-leader. However, this is a poor heuristic for predicting r-leader ligands; in fact, among r-leaders that regulate multi-gene operons in Escherichia coli or Bacillus subtilis, the ligand is most often not encoded by the immediately downstream gene (Additional file 1: Table S4) [7]. In early research, it was proposed that $r$-leader ligands are proteins that bind rRNA independently of other proteins [5]. Such primary-binding proteins have been distinguished from secondary-binding proteins, which bind rRNA only in the presence of other r-proteins [30, 31]. However, while primary-binding proteins are the most typical $r$-leader ligands, some subsequently validated $r$-leaders bind secondary-binding proteins, e.g., S2, S6:S18 and L25 [7, 30, 31].

We therefore exploited the observation that many r-leader ligands are common to multiple organisms, e.g., E. coli and B. subtilis [7] (Additional file 1: Table S4). We used this observation for motifs that regulate multiple genes where one of these genes encodes an r-protein that has been previously established as an r-leader's ligand. For such motifs, we predict that the previously established $r$-protein is also the ligand of the new motif. To date, no exception to this assumption has been experimentally established. However, there is no guarantee that this situation will hold for all cases in the future (Additional file 1: Supplementary text). Despite this caveat, the assumption presents the best currently known method to predict $r$-leader ligands.

For 10 of the remaining 11 motifs that regulate more than one ribosomal gene, exactly one of the regulated genes has previously been identified as the ligand of an r-leader in a distinct bacterial lineage (Table 1, "Ligand basis" is "Prior"). One motif remains that is upstream of multiple genes, none of which encode a previously established $r$-leader ligand. Our best guess for this motif's ligand is the immediately downstream gene, encoding L2 (Table 1).
It is important to emphasize that our predictions of ligands vary greatly in confidence, given the caveats mentioned in the preceding paragraphs. Because of the potential for incorrect ligand hypotheses, we list additional genes associated with the various motifs (Table 1 and Additional file 2). An additional point is that it is possible that some RNAs that regulate r-proteins in cis do not function by binding any r-protein [32].

Given a tentative ligand, we investigated possible mimicry of the rRNA by comparing the r-leader to the rRNA's protein binding site (see Methods). Because atomic-resolution structures of our r-leaders are not available, we conducted this analysis using conserved features in the sequence and secondary structure. We were particularly interested in rRNA nucleotides involved in the binding interface that are highly conserved, as these nucleotides and their structural contexts are most likely to be adopted by an r-leader in order to imitate the rRNA. In performing this analysis, it is important to consider the possibility that apparent similarities between one or two conserved nucleotides might arise by chance. We conducted these analyses manually (see Methods). For r-leaders regulating multiple genes, we analyzed both the protein encoded by the immediately downstream gene and the most-likely protein ligand, if these are different. The consideration of multiple potential ligands and associated rRNA regions increases the chances of finding spurious similarities in local secondary structures, and therefore we did not attempt a comparison of more than two proteins. Evidence of possible mimicry is presented where the similarity between the $r$ leader and rRNA is striking (see Methods).

In the following text, we discuss specific findings about the 20 novel r-leaders. Additional details are in Additional file 1: Supplementary text.

\section{L31 r-leaders: five motifs for one r-protein}

We found five motifs that likely bind the bacterial L31 rprotein (Table 1, Fig. 2), for which no r-leader has previously been identified. All five L31 motifs consist of a single hairpin, but the differing patterns of sequence conservation and bulge locations suggest that the motifs 


\begin{tabular}{|c|c|c|c|c|c|c|}
\hline 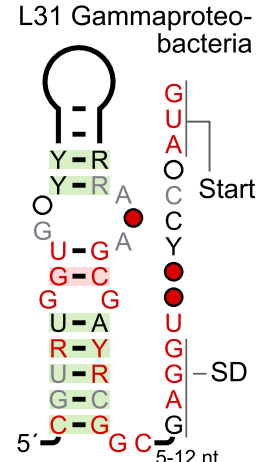 & 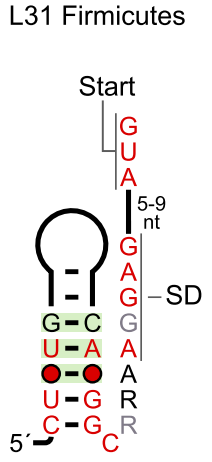 & 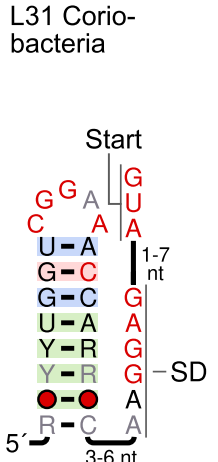 & 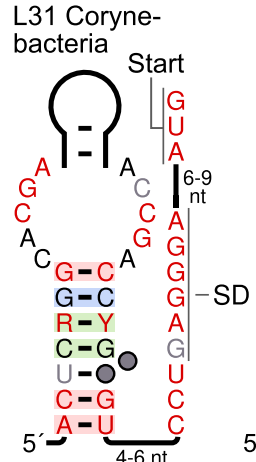 & 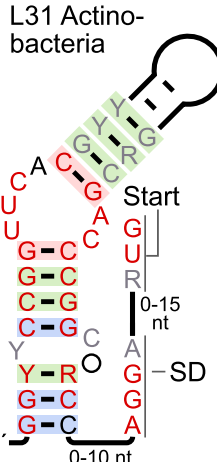 & 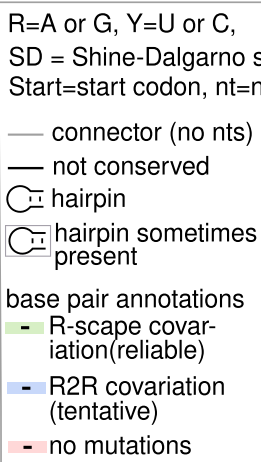 & $\begin{array}{l}\text { sequence, } \\
\text { nucleotides } \\
\text { nucleotide } \\
\text { identity } \\
\text { N } 97 \% \\
\text { N } 90 \% \\
\text { N } 75 \% \\
\text { nucleotide } \\
\text { present } \\
\text { O } 97 \% \\
\text { O } 90 \% \\
\text { O } 75 \% \\
\text { O } 50 \%\end{array}$ \\
\hline
\end{tabular}

Fig. 2 Conserved features of L31 r-leader motifs. The bacterial lineage is indicated. The inset explains annotations used throughout this paper, not all of which occur in this figure. Information on our analysis of covariation is in Methods. Only R-scape annotations are statistically reliable

are structurally unrelated. However, it is conceivable that elucidation of the binding determinants or an atomicresolution structure would reveal currently obscure similarities.

The predicted L31 r-leaders span multiple phyla, although each individual $r$-leader motif is restricted to one phylum. We found novel L31 r-leaders in E. coli and $B$. subtilis, which is surprising, given the extensive study of these organisms.

The precise structure and role of the L31 protein in the ribosome has been unclear [33, 34]. A truncated form of L31 is likely a side effect of ribosome purification methods, and may have contributed to confusing data about L31 function [33]. Recent results suggest that L31 is part of a bridge between the small and large subunits and interacts with 5S rRNA, 16S rRNA and rproteins L5, S13, S14 and S19 [34]. The ability of the $30 \mathrm{~S}$ head domain to swivel is accommodated via changes in the flexible structure of L31 and its intermolecular interactions $[34,35]$.

Many bacteria have two L31 genes, where one of these two genes encodes a zinc-binding protein containing the amino acid sequence CXXC, where $\mathrm{X}$ is any amino acid [36]. These paralogous genes were proposed to contribute to regulation of zinc homeostasis [36, 37]. It is conceivable that the L31-associated motifs regulate L31 genes as part of zinc homeostasis, and are not L31binding $r$-leaders. If this hypothesis is true, all five motifs would most likely have the same biochemical function, i.e., would either all regulate zinc-binding L31 proteins, or all regulate non-zinc-binding L31 proteins. However, this is not the case (Additional file 1: Supplementary text, Additional file 1: Table S5). Therefore, this hypothesis is probably incorrect, although we cannot rule out the possibility that some of the five L31 motifs have different biological functions from others. Curiously, we notice that most organisms contain zero or one L31 motif, for all five r-leader motifs (Additional file 2).
Given that many organisms have two L31 genes that could be regulated, we are uncertain as to why only one is associated with an r-leader.

\section{Imitation of the rRNA: L20, eL15, S15 and S4 r-leaders}

We found motifs that exhibit similarity to the rRNA's binding site for either the L20, eL15, S15 or S4 rproteins, and they are our best candidates for rRNA mimicry. These similarities are based on several conserved nucleotides in the rRNA binding site that resemble conserved r-leader nucleotides. Additional support derives in some cases from similarities between our $r$ leaders and previously published $r$-leaders whose rRNA mimicry was established in prior work.

R-leaders that bind L20 have been previously established in Gammaproteobacteria and Firmicutes [7]. These leaders each exhibit two conserved regions that correspond to two parts of the relevant binding site in the rRNA: two consecutive G-C base pairs and an AA dimer [7, 38] (Fig. 3a, Additional file 1: Figure S6). The two regions dock with each other in the ribosome (PDB model 4 V85). We found a candidate L20 r-leader in Deltaproteobacteria that appears to conserve the same two regions of the rRNA binding site of L20, but in yet another structural scaffold (Fig. 3a). In the new motif's AA dimer, one A nucleotide is predicted as pairing, but the existence of the base pair is unclear, as it is strictly conserved and therefore does not exhibit covariation. The two conserved elements are separated by a helix of several consecutive base pairs, which could bend to accommodate the docking interaction. Because of the apparent similarity to previously established L20 r-leaders that mimic the rRNA (Additional file 1: Figure S6), we suspect that the Deltaproteobacteria L20 motif also uses rRNA mimicry.

An r-leader motif that likely binds eL15 (the eukaryotic and archaeal L15 r-protein) (Fig. 3b) exhibits possible similarity to the eL15 binding site in the 

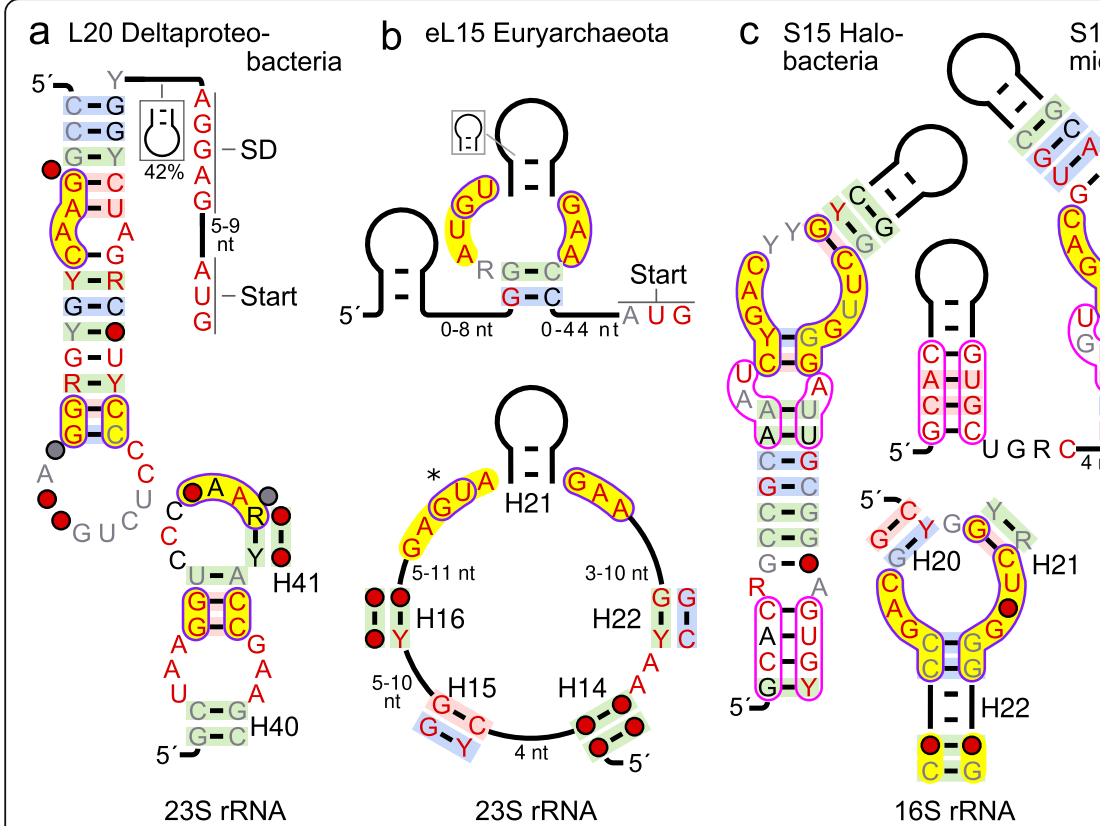

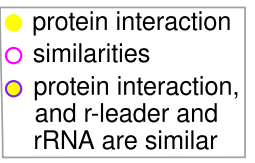

16S rRNA

d S4 Fusobacteriales microbia S15 Methano-

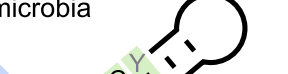

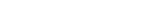

Fig. 3 Proposed rRNA imitation by r-leaders. Upper row: novel r-leader motifs. Lower row: rRNA binding site for the relevant r-protein. Annotations: as in Fig. 2. Yellow shading: nucleotides that interact with the relevant r-protein. Purple outline with yellow shading: interacting nucleotides that are similar in r-leader and rRNA. Purple outline: similar r-leader nucleotides that do not resemble the rRNA. H20-H40: Thermus thermophilus helix numbers from [39] or [40]. a L20 motif. b Archaeal motif for the eL15 protein. The asterisk marks a region of the bulged-G module that is only partially similar in the r-leader (see text). c S15 motifs in Halobacteria and Methanomicrobia. $\mathbf{d}$ S4 motif from Fusobacteriales

archaeal ribosome. The rRNA nucleotides that directly bind the eL15 protein (Fig. 3b) form a bulged-G module structure [41] (also called a Sarcin-Ricin loop or E-loop), a common structure in RNAs that is often associated with the binding of proteins [41]. The eL15 r-leader motif contains conserved nucleotides that are similar to a bulged-G module, but some nucleotides that are highly conserved in bulged-G modules are altered or missing in the r-leader motif (Fig. 3b, asterisk). Therefore, despite similar nucleotides, it is unclear whether this r-leader imitates the rRNA.

The strongest candidates for mimicry are two S15 rleaders. The S15 protein is the target of four experimentally confirmed and two predicted bacterial r-leaders [42]. Experimental analysis of the four validated S15 r-leaders revealed how RNA-protein interactions can evolve over large evolutionary distances in which the r-leaders evolve distinct-yet partially related-strategies to bind the S15 protein [15]. Each of these motifs mimics one of two sites in the rRNA: (1) stacked G-C, G-U base pairs, or (2) conserved nucleotides within a three-stem junction [7] (Fig. 3c, Additional file 1: Figure S10).

We found two related archaeal S15 r-leaders (Fig. 3c). Although their secondary structures differ, a part of each motif closely resembles the multistem junction in the rRNA, strongly suggesting imitation (Fig. 3c). In addition to their mimicry of the rRNA, the motifs have regions that resemble each other, but do not resemble the rRNA
(Fig. 3c, non-filled purple boundaries). One of these regions is a stem that occurs in different structural contexts in the two motifs. Given that the stem is conserved despite a structural rearrangement, it is likely that it is functionally important. However, the significance of the common regions to $\mathrm{r}$-protein binding or gene regulation is unclear. Regardless, the two archaeal S15 motifs expand the scope of studying the myriad r-leaders for this r-protein to the domain Archaea.

An S4 r-leader that likely mimics the rRNA is described in the next section.

\section{S4 r-leaders}

S4 is the experimentally confirmed target of known, structurally unrelated r-leaders in Gammaproteobacteria $[43,44]$ and Firmicutes [14, 45], although none are present in Clostridia, a class of Firmicutes [14]. The Gammaproteobacteria motif does not mimic the rRNA, but a conserved GUAA sequence in the Firmicutes motif was found to imitate the rRNA $[14,46]$.

Four of the novel $r$-leader motifs most likely bind the S4 r-protein (Table 1, Fig. 3d, Fig. 4a). We observed potential for the S4 motif in Fusobacteria to imitate the rRNA's binding site (Fig. 3d). The conserved GUAA sequence in the previously published Firmicutes r-leader (Additional file 1: Figure S8) is shared by the Fusobacterial motif. Moreover, the GUAA sequence occurs in the context of other structural and sequence similarities 


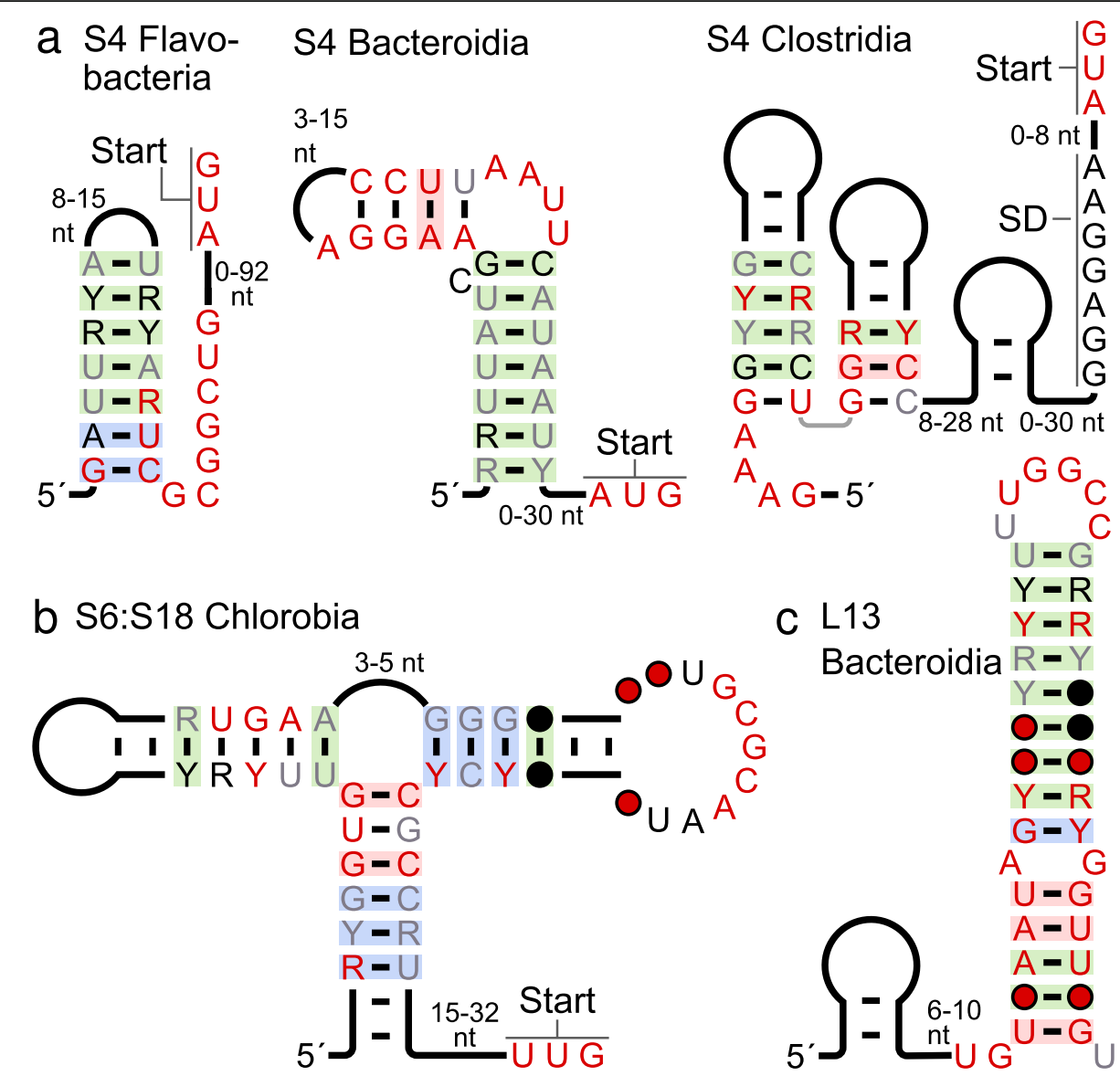

Fig. 4 S4, S6:S18 and L13 r-leader motifs. (All 20 motifs are shown in Additional file 1: Figures S2-S11). Annotations are as in Fig. 2. a S4 motifs that do not appear to imitate the rRNA. b S6:S18 motif in Chlorobi. c L13 in Bacteroidetes

between the two motifs, so this GUAA sequence in the Fusobacteria motif presumably also resembles the rRNA. A detailed comparison between the motifs, which also describes important differences between them, is in Additional file 1: Supplementary text.

The new S4 r-leader in Clostridia (Fig. 4a) has a conserved GAAA sequence ( $5^{\prime}$ end of molecule) that could resemble the aforementioned GUAA sequence, but this sequence is less similar to that of the rRNA. Therefore, it is unclear whether this r-leader might also imitate the rRNA. The two remaining S4 motifs, occurring in the related lineages Bacteroidia and Flavobacteria (Fig. 4a), exhibit no meaningful similarity to one another (other than the fact that they are both hairpins), to the previously published S4 r-leaders or to the rRNA.

\section{S6:S18 r-leader}

The S6 and S18 proteins function as a dimer [23, 24]. A previously known r-leader binding S6:S18 (Additional file 1: Figure S9) is very widespread in bacteria [23, 24], but missing in certain lineages such as the phylum
Chlorobi [24]. We found an r-leader (Table 1, Additional file 1: Figure S9) that most likely also binds S6:S18 and is restricted to the phylum Chlorobi (Fig. 4b). The widespread S6:S18 r-leader exhibits a clear similarity to the rRNA binding site (Additional file 1: Figure S9) and the relevant nucleotides are essential for binding the protein dimer [23, 24]. By contrast, the new Chlorobi version of this motif does not appear to resemble the rRNA.

\section{Archaeal r-leaders: S15, eL15, L4 r-leaders}

Four archaeal r-leaders are among the novel motifs (Table 1). Three, which are expected to bind the S15 or eL15 proteins, were discussed above. We noticed some poly-U stretches immediately after S15 r-leaders in Methanomicrobia (Additional files 2 and 3) that could correspond to intrinsic termination signals, which are still not well understood in archaea $[47,48]$. The fourth archaeal r-leader appears upstream of operons containing the L4 r-protein (Additional file 1: Figure S2, Supplementary text), which is the ligand of a previously established r-leader in bacteria [7]. 


\section{Other r-leaders}

We now note properties of the remaining candidate $r$ leaders beyond those summarized in Table 1. Some additional details are discussed in Additional file 1: Supplementary text. An experimentally verified L13 r-leader occurs in E. coli $[7,49]$, and a structurally distinct L13 rleader motif was previously predicted in Firmicutes [29]. We found a third, structurally unrelated L13 r-leader in Bacteroidetes (Fig. 4c).

A predicted L17 r-leader occurs in both Actinobacteria and Proteobacteria. This motif is the only candidate $r-$ leader we found that is clearly used by more than one phylum, suggesting that such widespread r-leaders are unusual among still-undiscovered r-leaders. A previously predicted RNA element occurring in distinct organisms and called the "L17DE motif" [25] is consistently found downstream of L17 genes, and could bind the encoded proteins or those of another gene in the upstream operon. The L17DE motif is restricted to the phylum Firmicutes.

\section{Discussion}

Using a comparative genomics approach, we found $20 \mathrm{r}$ leaders in multiple lineages of bacteria and archaea. These predictions are supported by covariation evidence that has proven reliable in past studies, and are in agreement with transcription start site (TSS) positions determined by high-throughput experiments. Nonetheless, experimental study of our candidates will be worthwhile to validate the predictions. Moreover, experimental analysis could lead to a better understanding of their biology and implications for RNA-protein interactions.

The identification of 20 predicted r-leaders represents an increase of more than $50 \%$ over the 35 previously published r-leaders (Additional file 1: Table S1) and suggests that many more r-leaders remain undiscovered in biology. Thus, further application of comparative genomics or other methods would likely uncover additional r-leaders. Additionally, our discovery of several r-leaders for $r$-proteins that were not previously known as $r$ leader ligands (i.e., L2, eL15, L17, L31, S16) suggests that current knowledge of what $r$-proteins can function as $r$ leader ligands is incomplete.

We found only one motif that spans more than one phylum. This agrees with analysis of Gammaproteobacteria suggesting that r-leaders are typically not widespread [44]. Since many phyla remain understudied, our results suggest that a significant number of $r$-leaders likely remain undiscovered in these other phyla. However, the L17 motif in Alphaproteobacteria and Actinobacteria shows that there is still room for the discovery of additional widespread r-leaders.

The four r-leader motifs in Archaea represent a significant increase in the number of known cis-regulatory
RNAs in these organisms. Archaea share many characteristics of both eukaryotes and bacteria [48]. The novel r-leaders present rare opportunities to learn about how cis-regulation works at the RNA level in Archaea, and, by extension, how transcription and translation processes may be co-opted for use in regulation.

The hypothesis that $r$-leaders will mimic rRNAs was presented soon after their discovery [5]. However, while many r-leaders do indeed imitate the rRNA binding site, several do not [7]. In the absence of rRNA imitation, distinct amino acids in the relevant $r$-proteins might be conserved that are not important for the r-protein's primary function. In this context, rRNA imitation might be an economical strategy that reduces the need for sequence conservation.

Of the 20 novel r-leader motifs, only $25 \%$ exhibit plausible similarities to the rRNA (Table 1, Fig. 3). By comparison, of 20 previously analyzed r-leaders [7], 50\% show good evidence of rRNA imitation [7]. This comparison could suggest that mimicry is, in fact, unusual in $r$-leaders, or that it is only common in widespread $r$ leader motifs, whereas the new r-leader motifs are generally restricted to one phylum. Another observation is that r-leaders of some proteins seem to consistently copy the rRNA, e.g., S15 and L20. Other proteins are inconsistent, e.g., the S6:S18 dimer has one previously published motif $[23,24]$ with clear similarity, and another (our Chlorobi motif) with no apparent similarity. Perhaps some protein-binding sites are more conducive to imitation than others.

We note that there are technical reasons that could account for our inability to establish a convincing similarity between r-leader and rRNA in some cases. First, comparisons between $r$-leaders and rRNA are more difficult because of uncertainty about the ligand and the absence of atomic-resolution structural information for the r-leaders. Second, in some cases, the rRNA binding sites show only a limited number of conserved nucleotides, or are not well conserved. In such cases, it is easy to find positions in the r-leader that might be similar, but difficult to establish that the similarity is meaningful. Therefore, it is possible that additional research will reveal more cases of structural mimicry among our r-leaders.

Previous work has already shown that there are $r$ proteins whose r-leaders exhibit multiple, distinct primary and secondary structures. Examples in E. coli and B. subtilis are the r-leaders for L20, S4 and S15 [7] (Additional file 1: Table S4). Considering all bacteria, four distinct r-leaders binding S15 were previously known [42]. Our work adds an archaeal version of S15, with two sub-types, and potential r-leaders for S4, L4, L13, L20, S4 and S6:S18 that add r-leaders to previously established protein ligands. Additionally, there are now five motifs associated with L31 genes. This plethora of 
solutions for binding a consistent protein could suggest that such interactions are easy to evolve, and creates fertile input for studies on the evolution of RNA-protein interactions [15].

\section{Conclusions}

This study presents 20 novel r-leaders in bacteria and archaea. The predictions are supported by covariation evidence, gene associations, and, in many cases, by highthroughput TSS experiments, and are thus a good starting point for detailed experimental investigation. With an increase of more than $50 \%$ in the number of known or predicted $r$-leaders, these results suggest that many more $r$-leaders remain undiscovered in bacteria and archaea.

R-leaders offer valuable opportunities to better understand RNA-protein interactions. The newly found $r$ leaders include multiple RNA structures that likely share a common ligand, or whose ligand is also the ligand of a previously established r-leader. Thus, the r-leaders represent alternate evolutionary solutions to the same protein-recognition problem. Additionally, some of the r-leaders exhibit a similar structure to that of the rRNA binding site, although for many r-leader motifs, no compelling similarity is apparent. The new r-leaders thus represent a foundation for different types of studies on r-leaders, gene regulation, ribosome assembly and RNAprotein interaction.

\section{Methods}

\section{Databases and software}

We used sequence data from the RefSeq nucleotide database version 72 [50] and metagenomic and metatranscriptomic data predominantly from IMG/M [51] and GenBank [52]. Genes were annotated as previously described [26]. Known RNAs were annotated using the Rfam Database [53] version 14.0 and papers on r-leaders $[7,14,23,29,42,44,49]$. Homology searches were conducted using Infernal version 1.1 [54], and alignments were analyzed for additional secondary structure using CMfinder version $0.4[26,55]$ and R-scape [56]. RNAs were drawn using R2R [57] and Inkscape [58]. Previously published analyses of transcriptomic data were used to determine whether our predicted r-leaders are consistent with information on transcription start sites (Additional file 1: Supplementary text).

\section{Covariation}

Several existing algorithms assess covariation in an alignment. Unfortunately, currently available computational approaches cannot consistently handle problems such as incorrect alignments, which can create misleading, invalid covariation. We therefore ultimately evaluated covariation manually. However, we used R-scape
[56] with the -s option as a guide. Assuming a valid alignment, R-scape is a statistically well-founded indicator of covariation. R-scape measures the statistical significance of a covariation signal using a random model of evolution that accounts for phylogenetic signals that can confound covariation analysis. Base pairs exhibiting statistically significant covariation are then reported. All 20 r-leader motifs exhibited statistically significant covariation according to $\mathrm{R}$-scape (Additional file 1: Table $\mathrm{S} 2)$. An issue with $\mathrm{R}$-scape is that it does not consider small covariation signals in multiple base pairs that together could provide compelling evidence of covariation. So, for the diagrams, we also depicted R2R's [57] simplistic and more permissive method to detect potential covariation. R2R reports covariation when there are at least two Watson-Crick or G-U base pairs among the sequences that differ at both positions and fewer than $10 \%$ of the sequences have non-canonical base pairs (i.e., base pairs that are neither Watson-Crick nor G-U) at those positions. Importantly, R2R's annotations are neither reliable nor statistically well-founded [57], and were therefore not used to draw conclusions about candidates. To allow manual or other analyses of our predicted $r$ leaders, we provide our alignments (see Availability of data and materials). Based on our manual analysis, we have not drawn stems we deem dubious, and note a few hairpins that are uncertain (Additional file 1: Supplementary text).

\section{Comparison of structures in rRNAs and r-leaders}

As part of the analysis of whether the new r-leaders structurally imitate rRNA, we used rRNA alignments available in the Rfam Database [53] version 14.0 (Additional file 1: Table S6). To more easily connect nucleotides in ribosomal crystal structures to alignment columns, the alignments were modified by adding rRNAs from the relevant crystal structure in Protein Databank (PDB) [59] using Infernal. Information on rRNA nucleotides that directly interact with a given $r$ protein were extracted from relevant studies (Additional file 1: Table S7) and inferred with the PyMOL (Schrödinger, LLC) version 2.0 function "Action: find > polar contacts $>$ to other atoms in object". We used a previously established crystal structure of the ribosome (PDB accession: 6GZQ) to conduct this analysis. For the eL15 r-leader, we used an archaeal ribosome (PDB accession: 1S72). Structural comparisons were performed manually based on drawings of conserved sequence and secondary structure features. We explored the use of CMCompare [60] to automatically find structural similarities, but found this approach unreliable, presumably due to the huge size of rRNAs and fact that the narrow phylogenetic distribution of many of our r-leaders results in an abundance of nucleotides that appear highly conserved, 
but are not of great biochemical or structural importance. Such misleading conservation impedes the detection of r-leader nucleotides likely to resemble the rRNA.

\section{Comparison of r-leader motifs}

We compared $\mathrm{r}$-leader motifs with previously established $r$-leaders and with each other, in order to determine if they were novel. As with comparisons to rRNA, we conducted these comparisons manually based on the conserved primary and secondary structural features of the relevant alignments. We paid particular attention to $r$ leaders that potentially had the same $r$-protein ligand, based on proteins encoded by regulated genes, as these r-leaders would be most likely to be similar.

\section{Operon analysis}

Information on regulated operons in Table 1 were estimated manually. Due to multiple factors, it is not straightforward to analyze this information automatically. Such complications include the high number of truncated contigs in metagenomic and shotgun sequences, errors in automated gene analysis (esp. the annotation of spurious genes) and natural variation in the distance between genes in operons, for example. Therefore, we initially assumed that co-transcribed genes can be up to 500 nucleotides apart, and considered only $\mathrm{r}$ leader homologs where at least $8 \mathrm{~kb}$ of sequence was available downstream of the RNA. We did not include genes that are consistently located far away from the previous gene, inconsistently positioned or often absent. Such additional genes that might be regulated are available in Additional file 2.

\section{Supplementary information}

Supplementary information accompanies this paper at https://doi.org/10. 1186/s12866-020-01823-6.

Additional file 1. Supplementary text, tables and figures.

Additional file 2. Alignments and information on downstream genes and taxonomy for all predicted r-leader motifs.

Additional file 3. Archive of machine-readable alignments for all rleader motifs that include flanking sequences and metadata. The alignments are stored in Stockholm format, which are text files that can be opened in any text editor (using a fixed-width font) and interpreted by software packages such as Infernal [54].

Additional file 4. Archive of machine-readable alignments for all rleader motifs that include only nucleotides within the predicted motifs, and do not include any metadata. The alignments are appropriate for tasks such as performing homology searches or re-analysis of our predicted secondary structures. The alignments are stored in Stockholm format.

\section{Abbreviations}

eL15: The eukaryotic- and archaeal-specific L15 ribosomal protein; PDB: Protein Data Bank; rRNA: Ribosomal RNA; r-protein: Ribosomal protein; r-leader: Ribosomal leader; UTR: Untranslated region; TSS: Transcription start site

\section{Acknowledgements}

We are grateful for computer time provided by the Center for Information Services and High-Performance Computing $(\mathrm{ZIH})$ at TU Dresden. We also thank Christina E. Weinberg for critical reading of the manuscript, and Michelle Meyer and bioinformaticians at Leipzig University for helpful comments.

\section{Authors' contributions}

ZW conceived of and supervised the study. IE conducted the study. Both authors wrote and approved the final manuscript.

\section{Funding}

This research was supported by the German Research Foundation (DFG) [WE6322/1-1 to Z.W.]. Funding for open access charge: Leipzig University and DFG via the Open Access Publishing program. These funding bodies played no role in the design of the study, in the collection, analysis or interpretation of data or in writing the manuscript.

\section{Availability of data and materials}

All data generated or analyzed during this study are included in this published article and its supplementary information files, or referenced therein. R-leader motif alignments are available (in addition to Additional files 2, 3 and 4) in an Rfam-Database-like [53] format at https://bitbucket.org/ zashaw/zashaweinbergdata/src/master/r-leader/, which is part of the repository at https://bitbucket.org/zashaw/zashaweinbergdata/src/master/.

\section{Ethics approval and consent to participate}

Not applicable.

\section{Consent for publication}

Not applicable.

\section{Competing interests}

The authors declare that they have no competing interests.

Received: 19 February 2020 Accepted: 14 May 2020

Published online: 24 May 2020

\section{References}

1. Lafontaine $\mathrm{DL}$, Tollervey D. The function and synthesis of ribosomes. Nat Rev Mol Cell Biol. 2001;2:514-20.

2. Steitz TA. A structural understanding of the dynamic ribosome machine. Nat Rev Mol Cell Biol. 2008;9:242-53.

3. Schmeing TM, Ramakrishnan V. What recent ribosome structures have revealed about the mechanism of translation. Nature. 2009;461:1234-42.

4. Nomura M, Gourse R, Baughman G. Regulation of the synthesis of ribosomes and ribosomal components. Annu Rev Biochem. 1984;53:75-117.

5. Nomura $M$, Yates $J$, Dean D, Post LE. Feedback regulation of ribosomal protein gene expression in Escherichia coli: structural homology of ribosomal RNA and ribosomal protein mRNA. Proc Natl Acad Sci U S A. 1980;77:7084-8.

6. Zengel JM, Lindahl L. Diverse mechanisms for regulating ribosomal protein synthesis in Escherichia coli. Prog Nucleic Acid Res Mol Biol. 1994;47:331-70.

7. Meyer MM. rRNA mimicry in RNA regulation of gene expression. Microbiol Spectr. 2018;6:RWR-0006-2017.

8. Fallon AM, Jinks CS, Strycharz GD, Nomura M. Regulation of ribosomal protein synthesis in Escherichia coli by selective mRNA inactivation. Proc Natl Acad Sci U S A. 1979;76:3411-5.

9. Olins PO, Nomura M. Translational regulation by ribosomal protein S8 in Escherichia coli: structural homology between rRNA binding site and feedback target on mRNA. Nucleic Acids Res. 1981;9:1757-64.

10. Mattheakis LC, Nomura M. Feedback regulation of the spc operon in Escherichia coli: translational coupling and mRNA processing. J Bacteriol. 1988;170:4484-92.

11. Draper DE. How do proteins recognize specific RNA sites? New clues from autogenously regulated ribosomal proteins. Trends Biochem Sci. 1989;14: 335-8.

12. Merianos $\mathrm{HJ}$. The structure of a ribosomal protein $\mathrm{S} 8 / \mathrm{spc}$ operon mRNA complex. RNA. 2004;10:954-64.

13. Nevskaya N, Tishchenko S, Gabdoulkhakov A, Nikonova E, Nikonov O, Nikulin A, et al. Ribosomal protein L1 recognizes the same specific structural 
motif in its target sites on the autoregulatory mRNA and 235 rRNA. Nucleic Acids Res. 2005;33:478-85.

14. Deiorio-Haggar K, Anthony J, Meyer MM. RNA structures regulating ribosomal protein biosynthesis in bacilli. RNA Biol. 2013;10:1180-4.

15. Slinger BL, Newman H, Lee Y, Pei S, Meyer MM. Co-evolution of bacterial ribosomal protein $\mathrm{S} 15$ with diverse mRNA regulatory structures. PLoS Genet. 2015;11:e1005720.

16. Toffano-Nioche C, Ott A, Crozat E, Nguyen AN, Zytnicki M, Leclerc F, et al. RNA at $92^{\circ} \mathrm{C}$ : the non-coding transcriptome of the hyperthermophilic archaeon Pyrococcus abyssi. RNA Biol. 2013;10:1211-20.

17. Speed MC, Burkhart BW, Picking JW, Santangelo TJ. An archaeal fluorideresponsive riboswitch provides an inducible expression system for hyperthermophiles. Appl Environ Microbiol. 2018. https://doi.org/10.1128/ AEM.02306-17

18. Pace NR, Thomas BC, Woese CR. Probing RNA structure, function, and history by comparative analysis. In: Gesteland RF, Cech TR, Atkins JF, editors. The RNA world. 2nd ed. Cold Spring Harbor: Cold Spring Harbor Laboratory Press; 1999. p. 113-41.

19. Gutell RR, Lee JC, Cannone JJ. The accuracy of ribosomal RNA comparative structure models. Curr Opin Struct Biol. 2002;12:301-10.

20. Michel F, Westhof E. Modelling of the three-dimensional architecture of group I catalytic introns based on comparative sequence analysis. J Mol Biol. 1990;216:585-610.

21. McCown PJ, Corbino KA, Stav S, Sherlock ME, Breaker RR. Riboswitch diversity and distribution. RNA. 2017;23:995-1011.

22. Lee K-Y, Lee B-J. Structural and biochemical properties of novel self-cleaving ribozymes. Molecules. 2017. https://doi.org/10.3390/molecules22040678

23. Fu Y, Deiorio-Haggar K, Soo MW, Meyer MM. Bacterial RNA motif in the 5' UTR of rpsF interacts with an S6:S18 complex. RNA. 2014;20:168-76.

24. Matelska D, Purta E, Panek S, Boniecki MJ, Bujnicki JM, Dunin-Horkawicz S. S6:S18 ribosomal protein complex interacts with a structural motif present in its own mRNA. RNA. 2013;19:1341-8.

25. Weinberg Z, Wang JX, Bogue J, Yang J, Corbino K, Moy RH, Breaker RR. Comparative genomics reveals 104 candidate structured RNAs from bacteria, archaea, and their metagenomes. Genome Biol. 2010;11:R31.

26. Weinberg Z, Lünse CE, Corbino KA, Ames TD, Nelson JW, Roth A, et al. Detection of 224 candidate structured RNAs by comparative analysis of specific subsets of intergenic regions. Nucleic Acids Res. 2017;45:10811-23.

27. Weinberg Z, Barrick JE, Yao Z, Roth A, Kim JN, Gore J, et al. Identification of 22 candidate structured RNAs in bacteria using the CMfinder comparative genomics pipeline. Nucleic Acids Res. 2007;35:4809-19.

28. Aseev LV, Bylinkina NS, Boni IV. Regulation of the rp/Y gene encoding $5 S$ rRNA binding protein L25 in Escherichia coli and related bacteria. RNA. 2015; 21:851-61.

29. Yao Z, Barrick J, Weinberg Z, Neph S, Breaker R, Tompa M, Ruzzo WL. A computational pipeline for high-throughput discovery of cis-regulatory noncoding RNA in prokaryotes. PLoS Comput Biol. 2007;3:e126.

30. Mulder AM, Yoshioka C, Beck AH, Bunner AE, Milligan RA, Potter CS, et al. Visualizing ribosome biogenesis: parallel assembly pathways for the 305 subunit. Science. 2010;330:673-7.

31. Davis JH, Tan YZ, Carragher B, Potter CS, Lyumkis D, Williamson JR. Modular assembly of the bacterial large ribosomal subunit. Cell. 2016;167:1610-1622. e15.

32. Aseev LV, Koledinskaya LS, Boni IV. Regulation of ribosomal protein operons rpIM-rpsl, rpmB-rpmG, and rp/U-rpmA at the transcriptional and translational levels. J Bacteriol. 2016;198:2494-502.

33. Ueta M, Wada C, Bessho Y, Maeda M, Wada A. Ribosomal protein L31 in Escherichia coli contributes to ribosome subunit association and translation, whereas short L31 cleaved by protease 7 reduces both activities. Genes Cells. 2017;22:452-71.

34. Liu Q, Fredrick K. Intersubunit bridges of the bacterial ribosome. J Mol Biol. 2016:428:2146-64.

35. Fischer N, Neumann P, Konevega AL, Bock LV, Ficner R, Rodnina MV, Stark $\mathrm{H}$. Structure of the E. coli ribosome-EF-Tu complex at $<3 \AA$ resolution by $\mathrm{C}_{\mathrm{s}}$ corrected cryo-EM. Nature. 2015;520:567-70.

36. Akanuma G, Nanamiya H, Natori Y, Nomura N, Kawamura F. Liberation of zinc-containing L31 (RpmE) from ribosomes by its paralogous gene product, YtiA, in Bacillus subtilis. J Bacteriol. 2006;188:2715-20.

37. Panina EM, Mironov AA, Gelfand MS. Comparative genomics of bacterial zinc regulons: enhanced ion transport, pathogenesis, and rearrangement of ribosomal proteins. Proc Natl Acad Sci U S A. 2003;100:9912-7.
38. Guillier M, Allemand F, Dardel F, Royer CA, Springer M, Chiaruttini C. Double molecular mimicry in Escherichia coli: binding of ribosomal protein L20 to its two sites in mRNA is similar to its binding to 235 rRNA. Mol Microbiol. 2005; 56:1441-56.

39. Thermus thermophilus small subunit ribosomal RNA. http://rna.ucsc.edu/ rnacenter/images/figs/thermus_16s_2ndry.pdf. Accessed 22 June 2019.

40. Thermus thermophilus large subunit ribosomal RNA. http://rna.ucsc.edu/ rnacenter/images/figs/thermus_23s_2ndry.pdf. Accessed 22 June 2019.

41. Leontis $N B$, Westhof $E$. A common motif organizes the structure of multihelix loops in 16 S and 23 S ribosomal RNAs. J Mol Biol. 1998;283:571-83.

42. Slinger BL, Deiorio-Haggar K, Anthony JS, Gilligan MM, Meyer MM. Discovery and validation of novel and distinct RNA regulators for ribosomal protein S15 in diverse bacterial phyla. BMC Genomics. 2014;15:657.

43. Deckman IC, Draper DE. Specific interaction between ribosomal protein $\$ 4$ and the a operon messenger RNA. Biochemistry. 1985;24:7860-5.

44. Fu Y, Deiorio-Haggar K, Anthony J, Meyer MM. Most RNAs regulating ribosomal protein biosynthesis in Escherichia coli are narrowly distributed to Gammaproteobacteria. Nucleic Acids Res. 2013;41:3491-503.

45. Grundy FJ, Henkin TM. The rpsD gene, encoding ribosomal protein S4, is autogenously regulated in Bacillus subtilis. J Bacteriol. 1991;173:4595-602.

46. Grundy FJ, Henkin TM. Characterization of the Bacillus subtilis rpsD regulatory target site. J Bacteriol. 1992;174:6763-70.

47. Maier L-K, Marchfelder A. It's all about the T: transcription termination in archaea. Biochem Soc Trans. 2019;47:461-8.

48. Blombach F, Matelska D, Fouqueau T, Cackett G, Werner F. Key concepts and challenges in archaeal transcription. J Mol Biol. 2019. https://doi.org/10. 1016/j.jmb.2019.06.020 .

49. Mustoe AM, Busan S, Rice GM, Hajdin CE, Peterson BK, Ruda VM, et al. Pervasive regulatory functions of mRNA structure revealed by highresolution SHAPE probing. Cell. 2018;173:181-195.e18.

50. O'Leary NA, Wright MW, Brister JR, Ciufo S, Haddad D, McVeigh R, et al. Reference sequence (RefSeq) database at NCBI: current status, taxonomic expansion, and functional annotation. Nucleic Acids Res. 2016;44:D733-45.

51. Chen I-MA, Chu K, Palaniappan K, Pillay M, Ratner A, Huang J, et al. IMG/M v.5.0: an integrated data management and comparative analysis system for microbial genomes and microbiomes. Nucleic Acids Res. 2019;47:D666-77.

52. Benson DA, Cavanaugh M, Clark K, Karsch-Mizrachi I, Lipman DJ, Ostell J Sayers EW. GenBank. Nucleic Acids Res. 2013;41:D36-42.

53. Kalvari I, Argasinska J, Quinones-Olvera N, Nawrocki EP, Rivas E, Eddy SR, et al. Rfam 13.0: shifting to a genome-centric resource for non-coding RNA families. Nucleic Acids Res. 2018;46:D335-42.

54. Nawrocki EP, Eddy SR. Infernal 1.1: 100-fold faster RNA homology searches. Bioinformatics. 2013;29:2933-5.

55. Yao Z, Weinberg Z, Ruzzo WL. CMfinder-a covariance model based RNA motif finding algorithm. Bioinformatics. 2006;22:445-52.

56. Rivas E, Clements J, Eddy SR. A statistical test for conserved RNA structure shows lack of evidence for structure in IncRNAs. Nat Methods. 2017;14:45-8.

57. Weinberg Z, Breaker RR. R2R-software to speed the depiction of aesthetic consensus RNA secondary structures. BMC Bioinformatics. 2011;12:3.

58. The Inkscape Project. http://www.inkscape.org. Accessed 1 May 2019.

59. Berman HM, Westbrook J, Feng Z, Gilliland G, Bhat TN, Weissig H, et al. The protein data Bank. Nucleic Acids Res. 2000;28:235-42.

60. Siederdissen $\mathrm{CH}$, Hofacker IL. Discriminatory power of RNA family models. Bioinformatics. 2010;26:1453-9.

\section{Publisher's Note}

Springer Nature remains neutral with regard to jurisdictional claims in published maps and institutional affiliations. 